\title{
Entity Name Data Type
}

National Cancer Institute

\section{Source}

National Cancer Institute. Entity Name Data Type. NCI Thesaurus. Code C95653.

A data type comprised of the name of an entity. 